\title{
The Adaptive Right Ventricle in Eisenmenger Syndrome: Potential Therapeutic Targets for Pulmonary Hypertension?
}

\author{
Rebecca Johnson Kameny, Sanjeev A. Datar, \\ Jason Boehme, and Jeffrey R. Fineman
}

\begin{abstract}
Pulmonary hypertension $(\mathrm{PH})$ is a rare disease with significant mortality despite targeted therapies. Among patients with $\mathrm{PH}$, both survival and functional class are more closely correlated with right ventricular (RV) function than with the degree of pulmonary artery pressure elevation or pulmonary vascular resistance. Unfortunately, the RV is usually exquisitely sensitive to increases in afterload associated with $\mathrm{PH}$, and progressive RV failure is the typical clinical course among most patients over time. However, in the subset of PH patients with congenital heart disease and Eisenmenger syndrome, survival is prolonged and RV function is preserved compared to patients with primary PH. This functional superiority may be due to a persistent RV fetal phenotype as patients continue to have a biventricular hypertrophy pattern which mimics fetal cardiac morphology. We have utilized an ovine model of congenital heart disease to demonstrate superior RV function following acute afterload stimulus-a unique RV Anrep effect. Further, we have shown shared gene expression patterns in the fetal and CHD model RV. In the future, understanding the underlying mechanisms of RV adaptation in CHD and PH may yield novel therapeutic strategies for all patients with pulmonary hypertension.
\end{abstract}

\section{Keywords}

Eisenmenger syndrome $\cdot$ Pulmonary hypertension $\cdot$ Right ventricle

\author{
R. J. Kameny · S. A. Datar · J. Boehme \\ Department of Pediatrics, University of California, San Francisco, San Francisco, CA, USA \\ J. R. Fineman $(\square)$ \\ Department of Pediatrics, University of California, San Francisco, San Francisco, CA, USA \\ Cardiovascular Research Institute, University of California, San Francisco, \\ San Francisco, CA, USA \\ e-mail: jeff.fineman@ucsf.edu \\ (C) The Editor(s) (if applicable) and The Author(s) 2020
}

T. Nakanishi et al. (eds.), Molecular Mechanism of Congenital Heart Disease

and Pulmonary Hypertension, https://doi.org/10.1007/978-981-15-1185-1_24 


\subsection{Introduction}

Pulmonary arterial hypertension $(\mathrm{PAH})$ is a rare but devastating disease that carries a significant burden of morbidity and mortality. Most investigations have focused on mechanisms that underlie abnormal pulmonary vascular reactivity and remodeling, which have led to the development of new therapies and improved survival. Similarly, much of the clinical evaluation of patients with PAH is based upon assessments of pulmonary arterial pressure and pulmonary vascular resistance. However, mortality is due to right ventricular (RV) failure, and there is growing recognition that indices of RV function in patients with $\mathrm{PAH}$-including right atrial pressure and cardiac index - are correlated most closely with not only survival but also functional class [1, 2]. Interestingly, patients with $\mathrm{PAH}$ associated with $\mathrm{CHD}$ have better functional capacity and survival than patients with other forms of PAH despite equivalent pulmonary artery pressures and advanced pulmonary arteriopathy [3]. However, the mechanisms that might account for this difference are unknown and have been the subject of limited investigation. Among patients with primary $\mathrm{PH}$, there is considerable variability in indices of RV function (cardiac output and right atrial pressure) relative to pulmonary artery pressure elevation. There is a subpopulation of patients who retain an adaptive phenotype with concentric hypertrophy and preserved mechanical efficiency; accordingly, these patients have improved survival compared to patients with a "maladaptive" RV phenotype [4]. A better understanding of preserved RV function in these patients might lead to novel therapeutic targets.

\subsection{Improved Survival in Eisenmenger Syndrome}

For decades, there has been a consistently observed survival advantage among patients with Eisenmenger syndrome compared to those with idiopathic $\mathrm{PH}$ or $\mathrm{PH}$ secondary to other causes [5]. Despite equivalent elevations in PA pressure and pulmonary vascular resistance compared with patients with IPAH, these patients have lower right atrial pressures and higher cardiac output. Moreover, at a similar level of PAP elevation, patients with ES have superior echocardiographic features of RV function [6]. Two major mechanisms for this observed survival benefit have been theorized. The presence of a communication at either the atrial or ventricular level (or both) allows right-to-left shunting of blood—a "pop-off"-preserving leftsided output during right ventricular failure. Another intriguing potential factor is persistence of a fetal RV phenotype due to the presence at birth of an unrestrictive post-tricuspid valve left-to-right shunt that applies systemic-level afterload to the $\mathrm{RV}$. In this situation, the RV does not undergo the deconditioning that normally occurs as pulmonary vascular resistance decreases after birth and, thereby, may be better suited to function against an increasing afterload as pulmonary hemodynamics worsen [5, 7]. Interestingly, a recent multicenter study of predictive factors of survival in patients with ES found that those with pre-tricuspid lesions had worse survival [8]. These data support the hypothesis that RV remodeling in the setting of high afterload associated with post-tricuspid CHD lesions confers additional benefit beyond right-to-left unloading through an intracardiac shunt. 


\subsection{Preserved Fetal Morphology in Eisenmenger Syndrome}

During fetal life, the right ventricle is the dominant ventricle, responsible for approximately two-thirds of combined cardiac output [9]. Commensurate with this function, the right and left ventricles are equally hypertrophied in fetal life; in normal physiology, RV hypertrophy regresses postnatally as the RV remodels into a highly efficient, compliant chamber which pumps a full cardiac output to the low resistance pulmonary vascular bed with approximately $1 / 5$ the energy expenditure of the left ventricle. In patients with posttricuspid lesions, the RV is continually exposed to high afterload and regression from fetal characteristics is attenuated or absent. Hopkins and Waggoner compared morphologic characteristics of 50 patients with Eisenmenger syndrome, infants with unrestrictive VSDs, and fetuses with normal anatomy; they found a consistent linear relationship between LV and RV wall thickness among these groups (Fig. 24.1) [10]. Furthermore, among patients with Eisenmenger syndrome, those with post-tricuspid (as opposed to

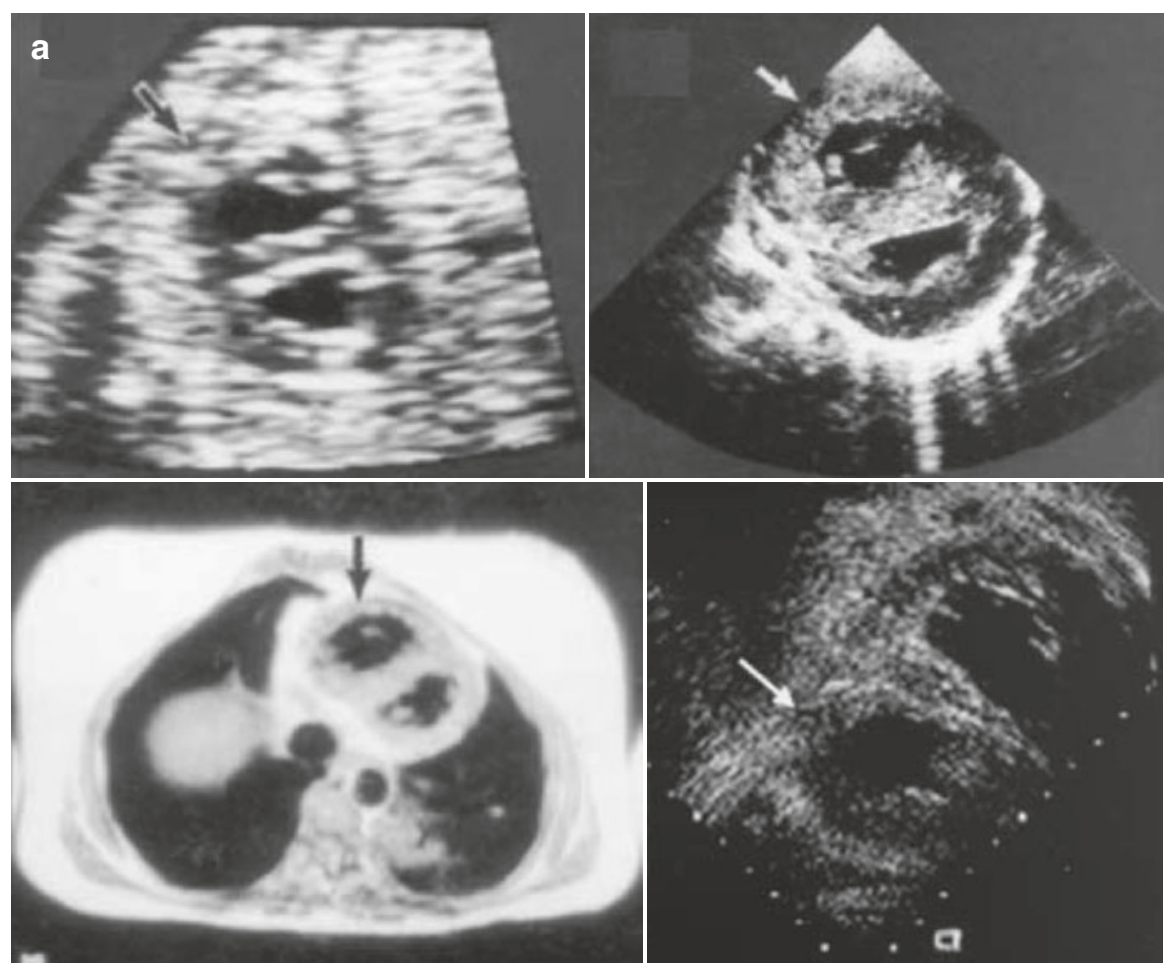

Fig. 24.1 Preserved fetal biventricular hypertrophy in patients with Eisenmenger syndrome (ES). Adapted from Hopkins and Waggoner. Severe pulmonary hypertension without right ventricular failure: the unique hearts of patients with Eisenmenger syndrome. AJC 2002. Reproduced with permission. (a) Cardiac imaging top left, normal 20-week fetal echo; top right, echo of 26-year old with truncus arteriosus and ES; bottom left, magnetic resonance imaging of 41-year old with patent ductus arteriosus and ES, 77-year old with perimembranous ventricular septal defect and ES. (b) Wall thickness. Linear Regression of LV and RV wall thickness in structurally normal fetal hearts, infants with unrestrictive ventricular septal defects, and adolescents and adults with unrestrictive post-tricuspid lesions and ES 


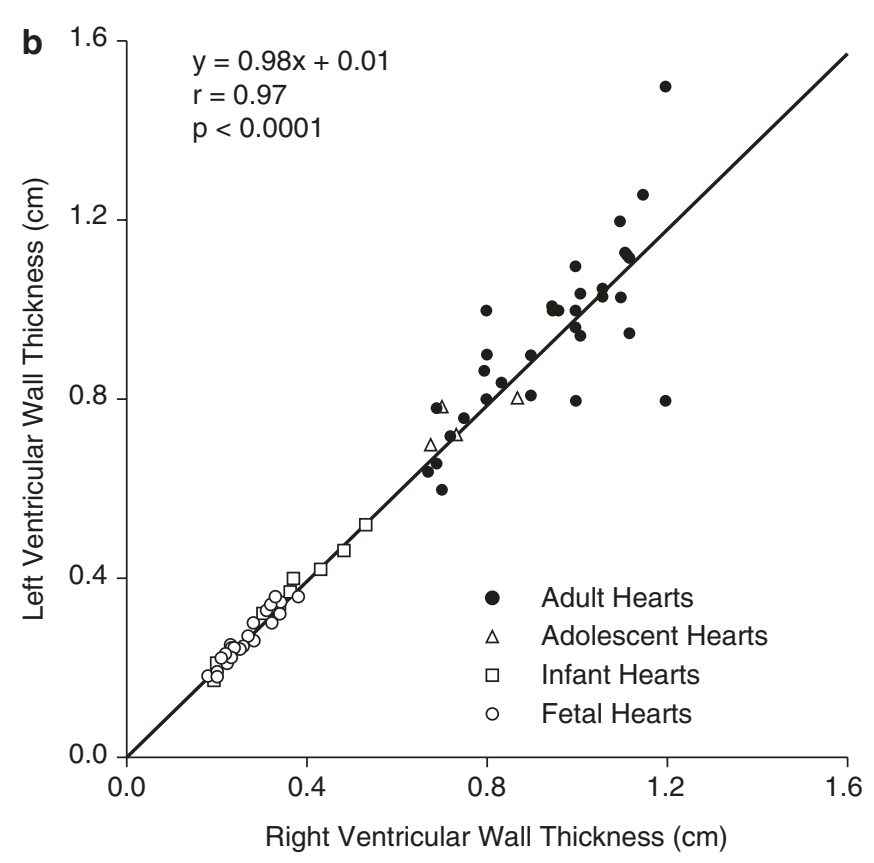

Fig. 24.1 (continued)

pre-tricuspid) lesions have better RV function and survival [11]. These findings suggest that post-tricuspid CHD lesions allow for continued postnatal exposure of the RV to systemic-level afterload, prevent the typical regression of RV hypertrophy postnatally, and condition the RV to respond to later increases in afterload associated with $\mathrm{PH}$.

\subsection{Fetal Phenotype in Ovine CHD Model}

Our laboratory has a well-established, clinical relevant model of CHD utilizing a large, fetally implanted aortopulmonary shunt, which continually exposes the RV to systemic level afterload during the perinatal transition and beyond (Fig. 24.2a) [12]. As a consequence of this hemodynamic stimulus, the RV in the juvenile shunt lamb is significantly hypertrophied compared to the control (Fig. 24.2b) [13]. In this CHD model, the $\mathrm{RV}$ of shunt lambs has many gene expression similarities with the RV of fetal lambs, including in $\alpha$ - and $\beta$-myosin heavy chain expression [13] and nitric oxide synthase expression and activity [14]. Further, we have utilized RNA sequencing to compare RV gene expression among fetal and juvenile shunt and control lambs. Eighty-two percent of genes that were differentially expressed in fetal RV tissue (compared to control) were also upregulated in shunt RV tissue (unpublished data). When subjected to gene ontology analysis, these 142 shared differentially expressed genes enrich a total of 25 biological pathways, including those associated with ECM remodeling, negative regulation of cellular proliferation, and cardiovascular system development—all pathways which might contribute to the observed adaptive RV phenotype. 


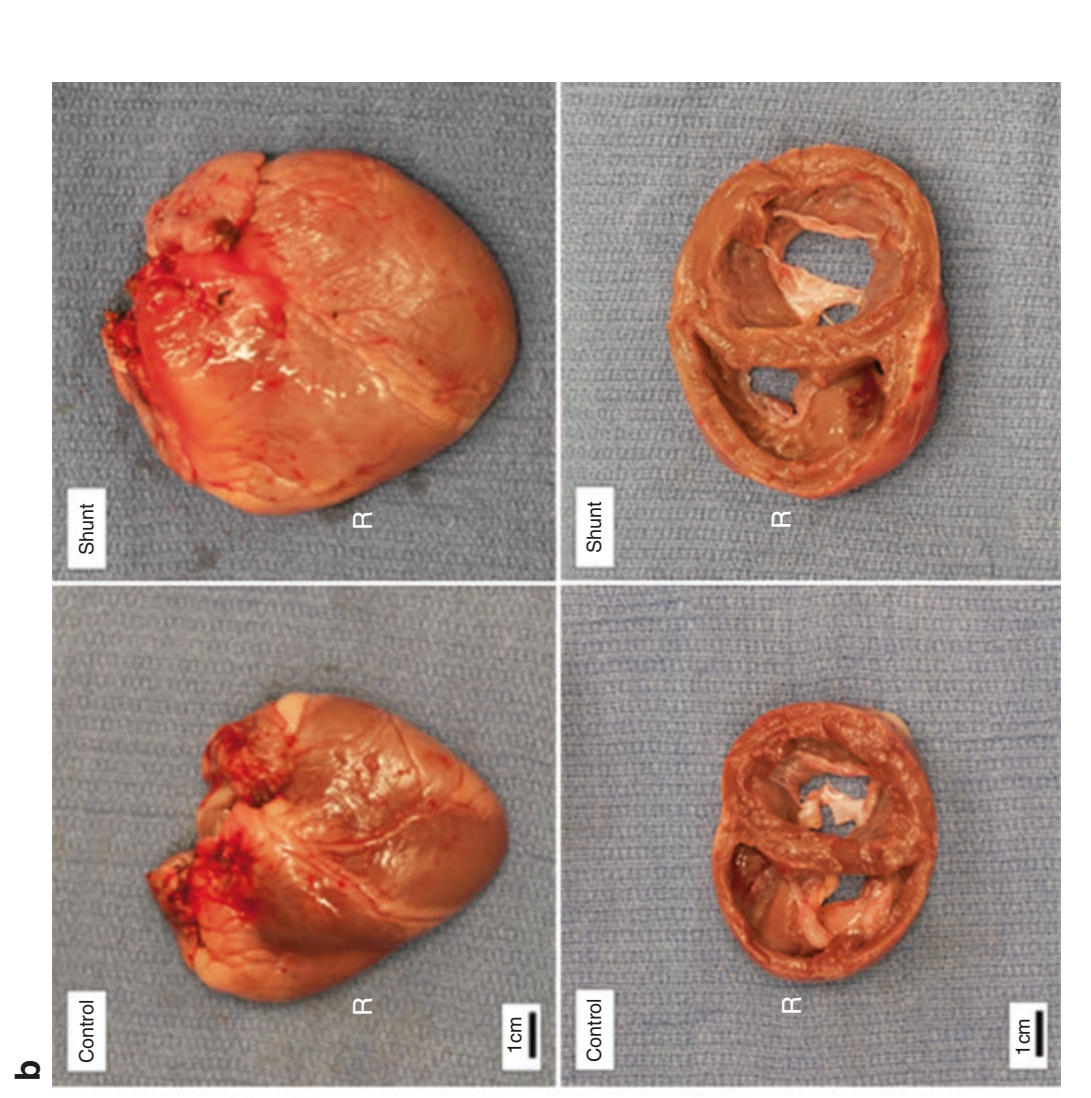

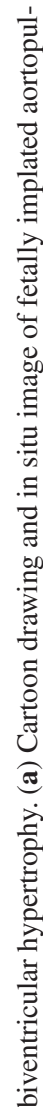
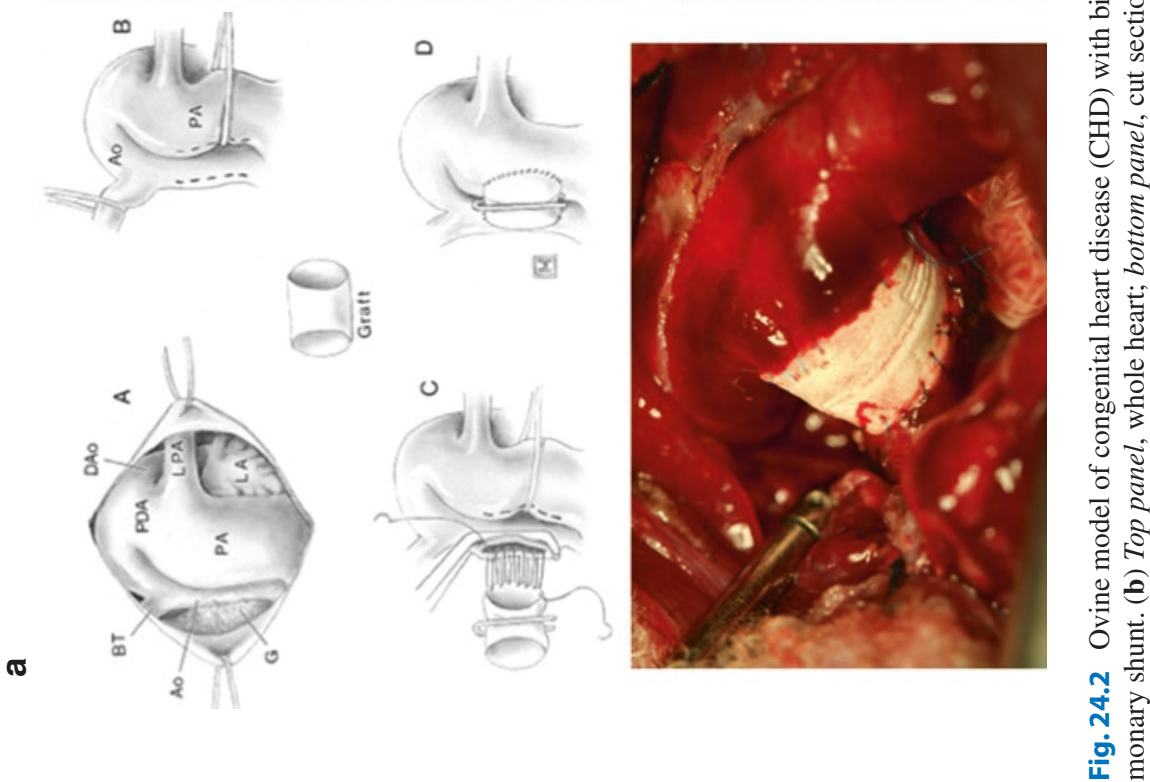

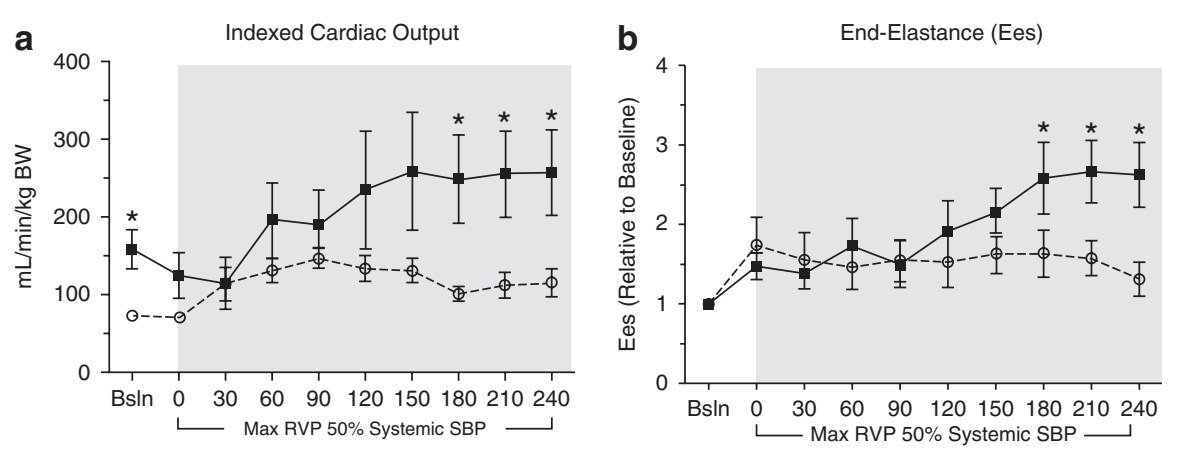

Fig. 24.3 Superior RV performance in ovine CHD model with acute RV afterload stimulus. Following $4 \mathrm{~h}$ of acute pulmonary artery banding to $50 \%$ of systemic pressure, (a) shunt lambs demonstrated continued superior cardiac index compared to controls and (b) shunt lambs increased $\mathrm{RV}$ contractility (Ees) by 2.5 -fold baseline

\subsection{The Adaptive RV Response to Acute Afterload-RV Anrep Effect}

In our model of CHD with chronic left-to-right shunting, we demonstrated that the RV of shunt lambs has an adaptive response compared to control RV when challenged with an acute afterload increase (imposed by pulmonary artery banding) with increased contractility and preservation of mechanical efficiency and ventricular-vascular coupling (Fig. 24.3). Our observation of a sustained increase in contractility in the shunt RV following afterload challenge is evidence of a novel Anrep effect, the second slow increase in contractility in response to myocyte stretch. Previously, this critical physiologic mechanism had only been observed in the left ventricle (LV) as the normal RV is exquisitely sensitive to increased afterload. Furthermore, after pulmonary artery banding, the RV in shunted animals had preserved ventricular-vascular coupling and ventricular efficiency relative to controls [13]. These adaptive physiologic responses to acutely increased afterload in shunted lambs may correlate with the response in patients with $\mathrm{CHD}$ and $\mathrm{PH}$.

\subsection{Potential Mechanisms of RV Anrep Effect}

Having established this novel Anrep effect, we next sought to understand potential underlying mechanisms. We first examined nitric oxide signaling as increased cardiac NO signaling has a variety of paracrine and autocrine effects which ultimately maximize cardiac function including improved excitationcontraction coupling, which is crucial for the Anrep effect [15]. We found a 


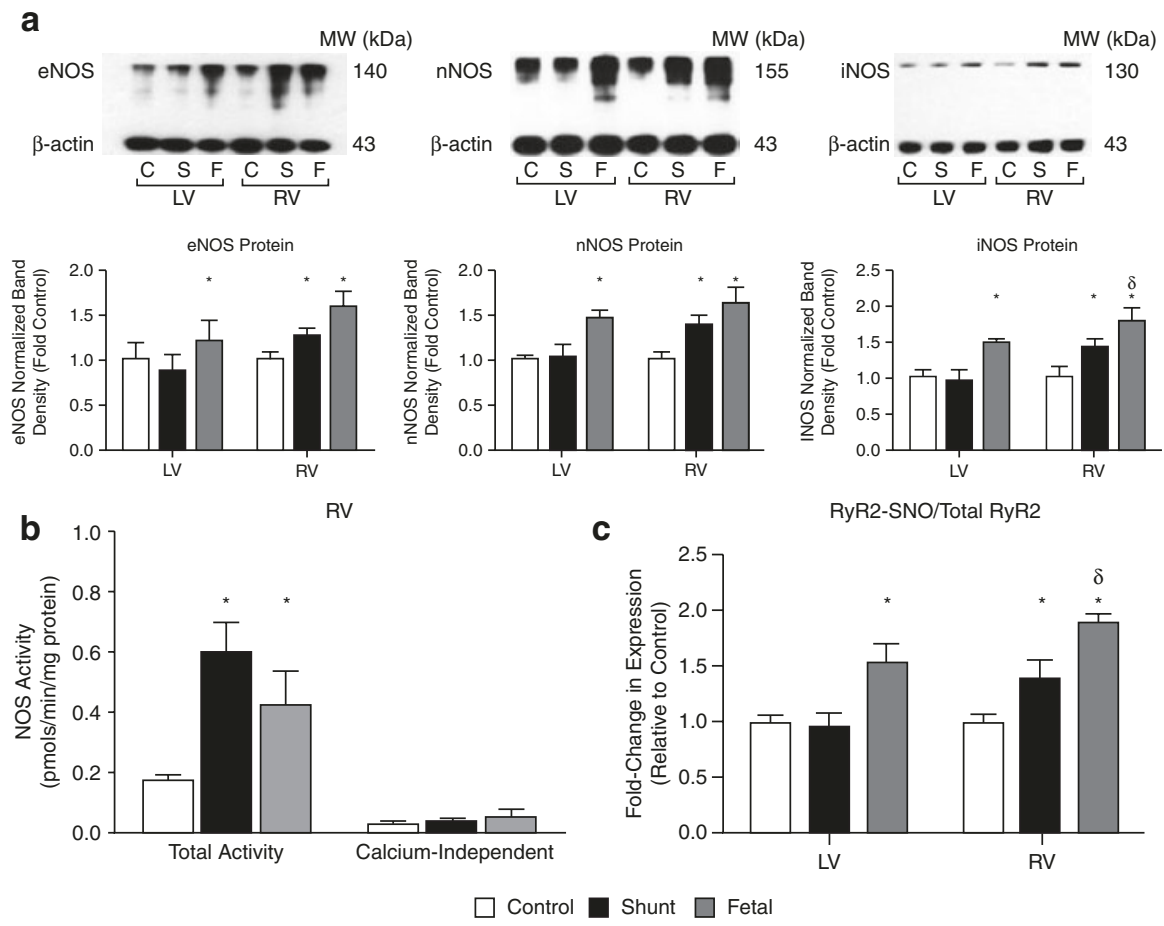

Fig. 24.4 RV nitric oxide signaling in ovine CHD model. (a) Increased expression of all nitric oxide synthase isoforms (eNOS, nNOS, and iNOS) in shunt and fetal RV. (b) Increased NOS activity in shunt and fetal RV, and (c) increased post-translational nitrosylation of ryanodine receptor 2 (RyR2) in shunt and fetal RV. Nitrosylation of RyR2 predisposes the open confirmation with resultant increased intracellular calcium and increased contractility

distinct shunt RV phenotype with consistently increased NOS expression, activity, and association of important cofactors for NOS activation; this distinct pattern was consistent in the RV of fetal and shunt but not control lambs (Fig. 24.4).

We next examined non-coding microRNA expression patterns as potential modulators of multiple pathways of gene expression [16]. We found important differences in miRNA expression in our adaptive RVH model compared human and animal maladaptive RVH with significant overlap in the fetus. Next, we investigated downstream effects of miR-199b and miR-29a expression as they relate to NFATcalcineurin mediated cardiac hypertrophy and fibrosis. Alterations in miR-199b and miR-29a and downstream effects are shown in Fig. 24.5 [17]. These changes yield valuable insight into one of the potential mechanisms behind adaptive RV hypertrophy seen in our model of ovine CHD. 


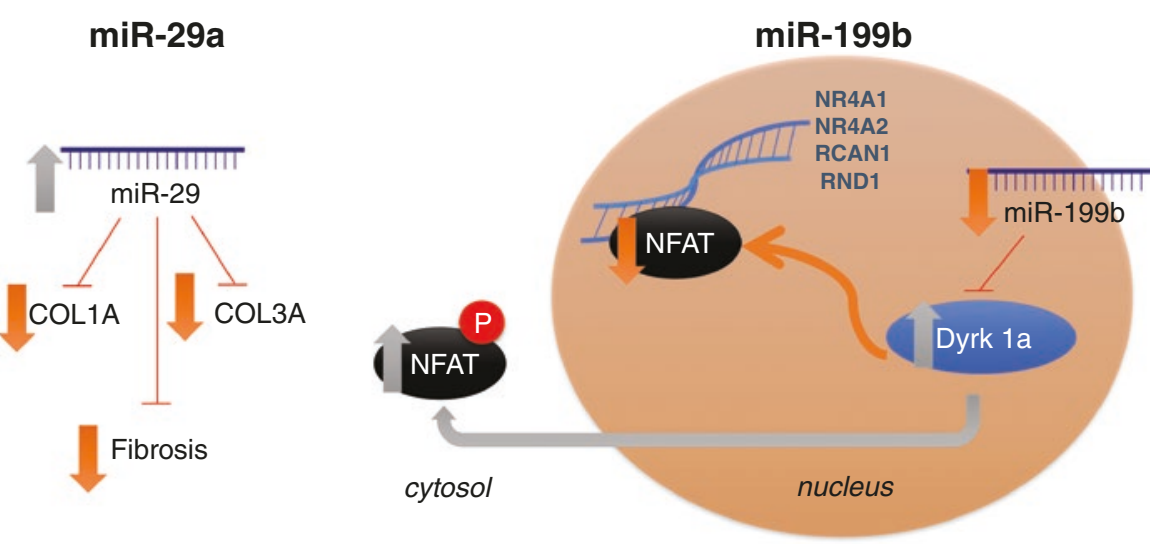

Fig. 24.5 miR 29 and 199b effects. Cartoon of downstream effects of observed changes in miR expression in shunt and fetal RV tissue. Increased miR-29 leads to decreased collagen 1 and 3 expression and decreased fibrosis. Decreased miR199b expression leads to increased Dyrk1a expression, which promotes NFAT phosphorylation and inhibits NFAT translocation to nucleus and transcription of NFAT/calcineurin-dependent heart failure gene program

\subsection{Future Directions and Clinical Implications}

Preserved RV function among patients with Eisenmenger Syndrome remains a clinical observation without a clearly identified pathophysiologic mechanism. Although a preserved RV fetal phenotype in these ES patients seems a logical conclusion based on both cardiovascular development and preliminary animal data, this hypothesis remains unproven. As the mechanisms for preserved RV function in these circumstances is better defined, the next step will be targeting of these pathways in all patients with preserved RV function in the hopes of improving the survival and functional status of these patients.

Ackowledgments This research was supported by grants from the National Institutes of Health (HL61284 to J.R.F. and 5T32HD049303-07 to J.R.F. and R.J.K.) and American Heart Association (14FTF19670001 to R.J.K.).

\section{References}

1. Voelkel NF, Quaife RA, Leinwand LA, et al. Right ventricular function and failure: report of a National Heart, Lung, and Blood Institute working group on cellular and molecular mechanisms of right heart failure. Circulation. 2006;114:1883-91. https://doi.org/10.1161/ CIRCULATIONAHA.106.632208.

2. Roche SL, Redington AN. The failing right ventricle in congenital heart disease. Can J Cardiol. 2013;29:768-78. https://doi.org/10.1016/j.cjca.2013.04.018. 
3. Hopkins WE, Ochoa LL, Richardson GW, Trulock EP. Comparison of the hemodynamics and survival of adults with severe primary pulmonary hypertension or Eisenmenger syndrome. $\mathrm{J}$ Heart Lung Transplant. 1996;15:100-5.

4. Simon MA, Deible C, Mathier MA, et al. Phenotyping the right ventricle in patients with pulmonary hypertension. Clin Transl Sci. 2009;2:294-9. https://doi. org/10.1111/j.1752-8062.2009.00134.x.

5. Hopkins WE. The remarkable right ventricle of patients with Eisenmenger syndrome. Coron Artery Dis. 2005;16:19-25.

6. Giusca S, Popa E, Amzulescu MS, et al. Is right ventricular remodeling in pulmonary hypertension dependent on etiology? An echocardiographic study. Echocardiography. 2016;33:54654. https://doi.org/10.1111/echo.13112.

7. Hopkins WE. Right ventricular performance in congenital heart disease: a physiologic and pathophysiologic perspective. Cardiol Clin. 2012;30:205-18. https://doi.org/10.1016/j. ccl.2012.03.006.

8. Kempny A, Hjortshøj CS, Gu H, et al. Predictors of death in contemporary adult patients with Eisenmenger syndrome: a multicenter study. Circulation. 2017;135:1432-40. https://doi. org/10.1161/CIRCULATIONAHA.116.023033.

9. Rudolph AM. Circulatory adjustments after birth: effects on ventricular septal defect. Br Heart J. 1971;33(Suppl):32-4.

10. Hopkins WE, Waggoner AD. Severe pulmonary hypertension without right ventricular failure: the unique hearts of patients with Eisenmenger syndrome. Am J Cardiol. 2002;89:34-8.

11. Alonso-Gonzalez R, Lopez-Guarch CJ, Subirana-Domenech MT, et al. Pulmonary hypertension and congenital heart disease: an insight from the REHAP National Registry. Int J Cardiol. 2015;184:717-23. https://doi.org/10.1016/j.ijcard.2015.02.031.

12. Reddy VM, Meyrick B, Wong J, et al. In utero placement of aortopulmonary shunts. A model of postnatal pulmonary hypertension with increased pulmonary blood flow in lambs. Circulation. 1995;92:606-13.

13. Johnson RC, Datar SA, Oishi PE, et al. Adaptive right ventricular performance in response to acutely increased afterload in a lamb model of congenital heart disease: evidence for enhanced Anrep effect. Am J Physiol Heart Circ Physiol. 2014;306(8):H1222-30.

14. Kameny RJ, He Y, Morris C, et al. Right ventricular nitric oxide signaling in an ovine model of congenital heart disease: a preserved fetal phenotype. Am J Physiol Heart Circ Physiol. 2015;309:H157-65. https://doi.org/10.1152/ajpheart.00103.2015.

15. Balligand JL, Feron O, Dessy C. eNOS activation by physical forces: from short-term regulation of contraction to chronic remodeling of cardiovascular tissues. Physiol Rev. 2009;89:481534. https://doi.org/10.1152/physrev.00042.2007.

16. Latronico MVG, Condorelli G. MicroRNAs and cardiac pathology. Nat Rev Cardiol. 2009;6:419-29. https://doi.org/10.1038/nrcardio.2009.56.

17. Kameny RJ, He Y, Zhu T, et al. Analysis of the microRNA signature driving adaptive right ventricular hypertrophy in an ovine model of congenital heart disease. Am J Physiol Heart Circ Physiol. 2018;315:H847-54. https://doi.org/10.1152/ajpheart.00057.2018. 
Open Access This chapter is licensed under the terms of the Creative Commons Attribution 4.0 International License (http://creativecommons.org/licenses/by/4.0/), which permits use, sharing, adaptation, distribution and reproduction in any medium or format, as long as you give appropriate credit to the original author(s) and the source, provide a link to the Creative Commons license and indicate if changes were made.

The images or other third party material in this chapter are included in the chapter's Creative Commons license, unless indicated otherwise in a credit line to the material. If material is not included in the chapter's Creative Commons license and your intended use is not permitted by statutory regulation or exceeds the permitted use, you will need to obtain permission directly from the copyright holder. 